Published in final edited form as:

Nat Rev Mol Cell Biol. 2009 February ; 10(2): 116-125. doi:10.1038/nrm2621.

\title{
MicroRNAs: key regulators of stem cells
}

\author{
Vamsi K. Gangaraju and Haifan Lin \\ Yale Stem Cell Center, Yale University School of Medicine, 10 Amistad Street, Room 220, New \\ Haven, Connecticut 06520-8002, USA
}

\section{Abstract}

The hallmark of a stem cell is its ability to self-renew and to produce numerous differentiated cells. This unique property is controlled by dynamic interplays between extrinsic signalling, epigenetic, transcriptional and post-transcriptional regulations. Recent research indicates that microRNAs (miRNAs) have an important role in regulating stem cell self-renewal and differentiation by repressing the translation of selected mRNAs in stem cells and differentiating daughter cells. Such a role has been shown in embryonic stem cells, germline stem cells and various somatic tissue stem cells. These findings reveal a new dimension of gene regulation in controlling stem cell fate and behaviour.

Tissue development and homeostasis are mediated by embryonic stem (ES) cells and tissue stem cells (also known as adult stem cells) ${ }^{1}$. ES cells are derived from the inner cell mass of the blastocyst-stage embryo and give rise to the fetus; in the process they generate tissue stem cells, progenitor cells and eventually every cell type in an organism. Tissue stem cells include somatic and germline stem cells, which develop, maintain and repair their resident tissues in developing and adult organisms. Both ES cells and tissue stem cells are capable of producing various types of differentiated cells and replicating themselves. Self-replicating ability is a hallmark property of stem cells and is generally termed self-renewal. Selfrenewal in tissue stem cells is often achieved by a unique pattern of asymmetric divisions. This generates one daughter cell that retains stem cell properties (self-renewal) and another daughter cell that is committed to specialized functions (differentiation) ${ }^{2}$. The self-renewing divisions of stem cells are controlled by both intercellular and intracellular mechanisms ${ }^{3}$. Whereas the intercellular mechanisms include signalling from the neighbouring niche cells,

(C) 2009 Macmillan Publishers Limited. All rights reserved

Correspondence to: H.F. Haifan.Lin@yale.edu.

DATABASES

Entrez Gene: http://www.ncbi.nlm.nih.gov/entrez/query.fcgi?db=gene

KIT

The miRNA registry: http://microrna.sanger.ac.uk/sequences let-7 | miR-1 | miR-15b | miR-16 | miR-17 | miR-20a | miR-21 | miR-22 | miR-23 | miR-24 | miR-26a | miR-29 | miR-106a | miR-124 | miR-125b | miR-128 | miR-133 | miR-134 | miR-144 | miR-150 | miR-155 | miR-181 | miR-203 | miR-206 | miR-221 | miR-222 | miR-223 | miR-290 | miR-296 | miR-301 | miR-302 | miR-424 | miR-451 | miR-470

UniProtKB: http://www.uniprot.org ALK4 | AML1 | BMP4 | DGCR8 | Dicer | Drosha | exportin 5 | EZH2 | FOG1 | GATA1 | HNF4A | LIN28 | nanog | OCT4 | REST | REX1 | SOX2 | SRF

FURTHER INFORMATION

Haifan Lin's homepage: http://www.cellbiology.yale.edu/faculty/lin_h/lin_h.html

ALL LINKS ARE ACTIVE IN THE ONLINE PDF 
intracellular mechanisms include differential gene expression that is controlled at epigenetic, transcriptional, translational and post-translational levels. Recently, microRNAs (miRNAs) have emerged as important players in translational regulation and have implications in controlling stem cell fate and behaviour ${ }^{4}$.

miRNAs are 20-25 nucleotide (nt) non-coding RNAs that bind to the $3^{\prime}$ untranslated region (UTR) of target mRNAs through an imperfect match to repress their translation and stability $^{5}$ (BOX 1). This is achieved by forming a ribonucleoprotein complex, called the RNA-induced silencing complex (RISC), that contains an Argonaute family member.

Occasionally, miRNAs have also been observed to activate target mRNA translation and to regulate their stability ${ }^{6}$. miRNAs are derived from precursor transcripts - called primary miRNAs (pri-miRNAs) - that are first processed in the nucleus into an intermediate form (pre-miRNAs) by the Microprocessor protein complex; this contains the Drosha and DiGeorge syndrome critical region gene 8 (DGCR8; also known as Pasha) proteins. The pre-miRNAs are then translocated by the exportin 5-RanGTP shuttle system into the cytoplasm, in which they are further processed by Dicer, an RNase III-like enzyme, into mature miRNAs. Recently, miRNAs have also been shown to be produced by two noncanonical, Drosha- or Dicer-dependent pathways. In the first pathway, the early processing step is done by spliceosome and a debranching enzyme that yields a short hairpin that is ready for further processing by Dicer. These non-canonical miRNAs have been termed mirtrons ${ }^{7,8}$. In the second pathway, short hairpin RNAs (shRNAs) are processed by unknown nucleases into pre-miRNAs and are further processed into miRNAs by Dicer. miRNAs that are derived in this way have been termed endogenous shRNA-derived miRNAs 9 .

\section{Box 1}

Biogenesis and regulatory features of the miRNA, siRNA and piRNA pathways

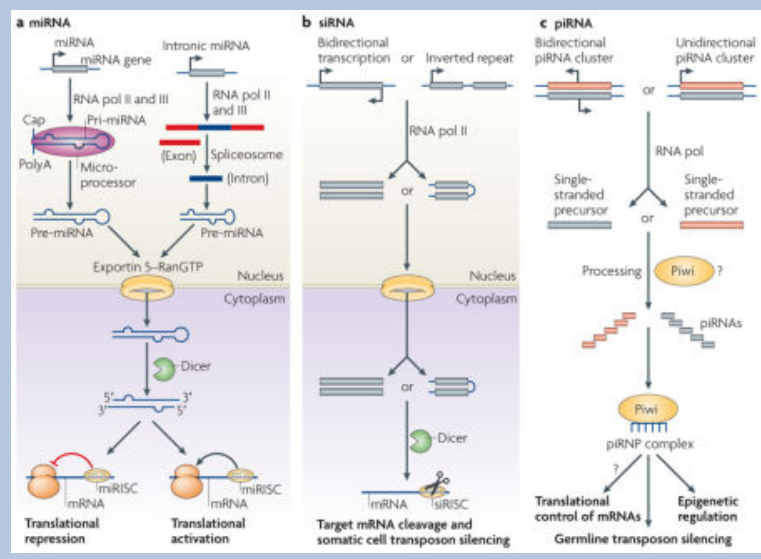

MicroRNA (miRNA) genes are transcribed by RNA polymerases II and III into primary transcripts called the pri-miRNAs (see the figure, panel a). These are processed into premiRNAs in the nucleus by a Microprocessor complex, which contains the RNase III enzyme Drosha and the double-stranded RNA-binding protein DiGeorge syndrome 
critical region gene 8 (DGCR8; also known as Pasha). Some miRNAs, called mirtrons, have also been shown to be generated from introns that bypass Drosha requirement. PremiRNAs are then transported by exportin 5, which is a karyopherin, and RanGTP into the cytoplasm, where they are further processed by the RNAse III enzyme Dicer. This results in double-stranded 20-25 nucleotide (nt) intermediates with 2 nt overhangs on the $3^{\prime}$ end. One of the RNA strands is then loaded by Dicer into an RNA-induced silencing complex (RISC), which contains an Argonaute-subfamily member, that then targets the $3^{\prime}$ untranslated region of the target mRNAs by an imperfect match between the miRNA and the mRNA, to repress translation. However, it has been recently shown that miRNAs can activate translation of target mRNAs ${ }^{6}$.

The small interfering RNA (siRNA) pathway initiates with either bidirectional transcription or transcription of an inverted repeat that results in a double-stranded precursor. This precursor is also processed in the cytoplasm by Dicer and is loaded into a small interfering RISC (siRISC) complex intended for target mRNA degradation (see the figure, panel b).

The Piwi-interacting RNA (piRNA) pathway seems to be particularly active in the germ line and functions in transposon silencing and epigenetic regulation. The exact mechanism of biogenesis is still unclear. Presumably, piRNAs originate from a long single-stranded RNA polymerase transcript (1-100 kilobases) that is often transcribed from a bidirectional piRNA cluster (see the figure, panel c). The precursor is subsequently processed by unknown mechanisms, which probably involve Piwi proteins but not Dicer, into mature piRNAs of 23-32 nt in length.

Two new classes of small non-coding RNAs have recently also been identified — the endogenous small interfering RNAs (endo-siRNAs) and the Piwi-interacting RNAs (piRNAs) (BOX 1). The biogenesis of endo-siRNAs initiates with either bidirectional transcription or the transcription of an inverted repeat. The resulting double- stranded RNA or the hairpin RNA precursors is then transferred into the cytoplasm, processed by Dicer and loaded into a RISC complex in a similar manner to the miRNA pathway. The small interfering RISC complexes then target mRNAs with complete complementarity to the loaded siRNAs and mediate their degradation ${ }^{10}$.

Whereas miRNA and siRNA production is generally Dicer dependent, piRNAs are generated from a long single-stranded precursor — probably by Piwi proteins but not by Dicer. piRNAs also differ from miRNAs and siRNAs in that they are usually 26-31 nt long, they associate with Piwi-subfamily proteins and map mostly to the repetitive regions of the genome $^{11}$. It is thought that piRNAs target transposons, and evidence is also available for epigenetic regulation in Drosophila melanogaster ${ }^{12}$. piRNAs might be important for stem cell self-renewal because their partner Piwi proteins are required for stem cell maintenance. Furthermore, a piRNA that was derived from a sub-telomeric region in D. melanogaster has been directly connected to germline stem cell self-renewal ${ }^{12}$. A brief description and comparison of these pathways is provided in BOX 1. 
Recently, the stem cell and miRNA fields have converged with the identification of stemcell-specific miRNAs ${ }^{13}$. In addition to canonical miRNAs, mirtrons and shRNA-derived miRNAs have also been identified in mouse ES cells ${ }^{9}$. It is now clear that miRNAs provide a new dimension to the regulation of stem cell functions. Based on their function in translational attenuation, miRNAs seem to regulate stem cell fate and behaviour by finetuning the protein levels of various factors that are required for stem cell or niche cell functions. In this article, we focus on current progress in understanding the biological function of miRNAs in ES cells and various tissue stem cells, with emphasis on their role in self-renewal and differentiation of stem cells.

\section{miRNA function in ES cells}

The ES cell system has been the main model for studying the function of miRNAs in stem cells, and, consequently, provides the most comprehensive knowledge on the subject.

\section{Overall role of the miRNA pathway}

The overall function of the miRNA pathway in ES cells has been evaluated in humans and mice by analysing the phenotypes of DGCR8 and Dicer mutants, respectively, as these two proteins have crucial roles in the production of mature miRNAs. In humans, DGCR8 (a double-stranded RNA-binding protein) partners with Drosha (an RNAse III enzyme) in the nucleus, and cleaves pri-miRNAs into $\sim 70 \mathrm{nt}$ pre-miRNA structures. These contain a $2 \mathrm{nt}$ overhang at the $3^{\prime}$ end. Pre-miRNAs are then translocated into the cytoplasm by exportin 5RanGTP and are further processed by Dicer to generate functional miRNAs ${ }^{14}$ (BOX 1). DGCR8 is exclusively involved in the miRNA pathway, whereas Dicer is involved in both miRNA and siRNA pathways. Therefore, the phenotype of Dicer mutants can be due to the absence of either miRNAs and/or siRNAs.

Dicer is essential for early mouse development, as its absence leads to embryonic lethality ${ }^{15}$. However, embryonic lethality does not seem to be due to the absence of ES cells, as Dicernull mouse ES cells are viable, although they exhibit severe growth and differentiation defects and prolonged G1 and G0 phases of the cell cycle. It is not clear whether cell cycle regulation is required for stem cell differentiation, or whether the delayed kinetics of cell cycle progression is a cellular response to other defects that are caused by the absence of Dicer and/or miRNAs. In vitro, Dicer-null ES cells fail to express differentiation markers, such as hepatocyte nuclear factor 4A (HNF4A; which is endodermal) and brachyury, bone morphogenetic protein 4 (BMP4) and GATA1 (which are mesodermal), even after the induction of differentiation. This shows that miRNAs and/or endo-siRNAs might function in ES cell differentiation ${ }^{16}$. In reality, this differentiation defect can probably be attributed solely to the absence of miRNAs, because a related study revealed that only the profiles of miRNAs in ES cells, but not of other small RNAs, change in the absence of Dicer ${ }^{17}$. This study also revealed that one important function of miRNAs in ES cells is to regulate cell cycle progression, because almost one-half of the 110,000 miRNA molecules that have been detected are produced by 4 miRNA loci (miR-21, the miR-17-92 cluster, the miR-15b-16 cluster and the miR-290-295 cluster) that regulate cell cycle progression and oncogenesis. 
Similar to the Dicer-null embryonic stem cells, DGCR8-deficient ES cells exhibit either delayed or reduced expression of differentiation markers, as well as delayed kinetics of cell cycle progression ${ }^{18}$. Most DGCR8-deficient ES cells are arrested in the G1 phase, which indicates that the main function of the miRNA pathway is to promote the ES cell cycle at the G1-S-phase transition. In addition, DGCR8-null ES cells exhibit differentiation defects as they fail to stably silence the expression of self-renewal markers, such as OCT4 (also known as OCT3 and POU5F1), REX1 (also known as ZFP42), nanog and SOX2 (REF. 18). In support of this, injected DGCR8-mutant ES cells into host mice fail to differentiate into different germ layers, in contrast with injected wild-type ES cells that can give rise to teratomas - tumours that can give rise to cell types of all three germ layers ${ }^{18}$. The similar phenotype of DGCR8 and Dicer mutants further confirms that Dicer in ES cells functions mainly in the miRNA pathway and that the predominant function of miRNAs is to regulate cell cycle progression during stem cell differentiation.

\section{ES cells have distinct miRNA signatures}

Whereas the Dicer and DGCR8 ES cell mutants provide an overall assessment of the function of the miRNA pathway in ES cells, cloning and deep sequencing of miRNAs from stem cells have revealed the identity of the specific miRNAs that are expressed in ES cells and might function in self-renewal and differentiation of ES cells. For example, in mice, the cloning and sequencing of small RNAs using conventional methods (BOX 2) revealed that the miR-290-295 cluster and miR-296 are specific to ES cells and that their levels decrease as the stem cells differentiate. In a simplified way, this indicates that the miR-290-295 cluster has specific roles in maintaining pluripotency ${ }^{13}$. However, more recent studies indicate that the real role of miR-290-295 is to induce differentiation (see below and FIG. 1). By contrast, levels of miR-21 and miR-22 increase substantially following the induction of differentiation, which indicates that these miRNAs might have important roles in stem cell differentiation (see TABLE 1 for a list of miRNAs that have been identified in various types of stem cell lineages). A similar study in human ES cells also shows that pluripotent stem cells have unique miRNAs, the levels of which decrease following differentiation ${ }^{19}$, even though no miRNA correlated to human ES cell differentiation has been identified. The functions of the ES cell-related miRNAs are being explored. 


\section{Box 2}

\section{DNA sequencing techniques for identifying small RNAs}

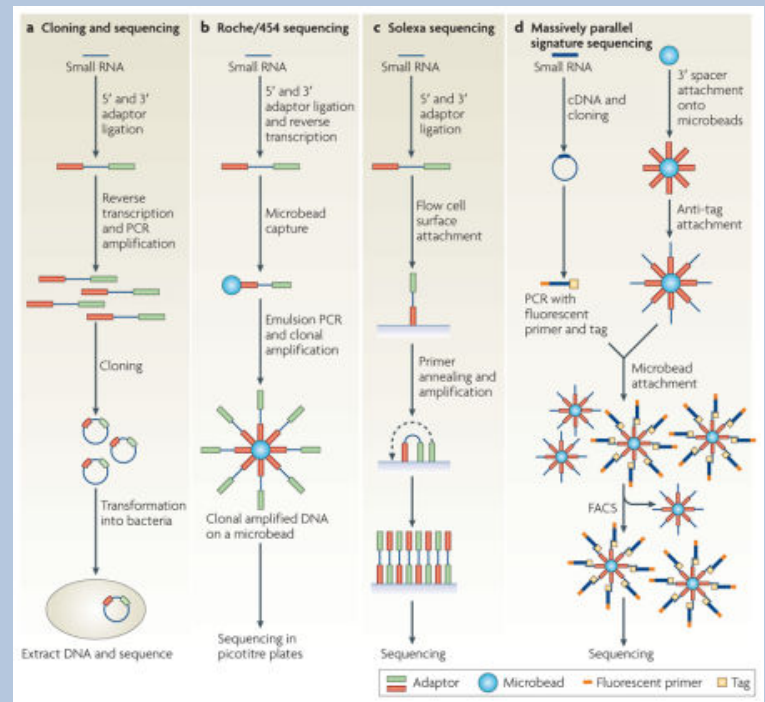

The past few years have seen the emergence of new techniques for the identification of small RNAs. Cloning and sequencing (see the figure, panel a), which was the first technique developed, involves the isolation of small RNA, the ligation of $5^{\prime}$ and $3^{\prime}$ adaptors, reverse transcription followed by PCR amplification, concatemerization and cloning into plasmids. These plasmids are then transformed into bacteria and each plasmid is then sequenced.

Roche/454 sequencing (see the figure, panel b) involves adaptor ligation (both $5^{\prime}$ and $3^{\prime}$ ) to small RNA, reverse transcription and then capturing onto microbeads, followed by emulsion PCR. In this process, PCR is performed in an emulsion droplet that contains a single bead with a template, which results in clonal amplification of the DNA. The DNA that is attached to the beads is then sequenced in picotitre plates using the Roche GS20 sequencer, which generates up to 2 million reads of 100-nucleotide (nt)-long molecules. However, the new FLX system from 454 Life Systems and Roche Applied Science can read 200-300-nt-long molecules.

In Solexa sequencing (see the figure, panel c), adaptors (both $5^{\prime}$ and $3^{\prime}$ ) are initially ligated onto small RNAs and then reverse transcribed and attached onto flow cell surface, followed by amplification (dotted arrow). This results in $\sim 1,000$ copies of the same template, which are then sequenced using four-colour 'sequencing-by-synthesis' technology (sequencing during the polymerase reaction on a solid surface), which generates up to 40 million reads of $<36 \mathrm{nt}$.

Massively parallel signature sequencing (MPSS) (see the figure, panel d) initiates with cloning a cDNA library of RNAs into plasmids, and PCR amplifying the cloned cDNAs with fluorescent primer on the $5^{\prime}$ end and 'tag' on the $3^{\prime}$ end. These are then annealed onto microbeads that are pre-coated with $3^{\prime}$ spacers and anti-tags (not shown). The smallcDNA-annealed beads are then sorted using fluorescence-activated cell sorting (FACS) 
and later sequenced. Since there is no PCR step involved, MPSS gives an additional advantage of measuring the expression level of small RNAs. Originally used for mRNAs, this method has been adapted for small RNAs.

Whereas a few of the identified miRNAs are conserved between human and mouse ES cells (miR-296, miR-301 and miR-302), a large proportion of the identified miRNAs are unique to human ES cells. The small overlap between the two data sets might reflect the fact that human and mouse ES cells are not found at the equivalent developmental stage. In addition, the small overlap seems to be partly due to the fact that the complete repertoire of miRNAs in stem cells has not been revealed in either species, and evolutionary differences are unlikely to be a major contributing factor. In support of this, a recent miRNA sequencing effort using the Solexa sequencing technology (BOX 2) discovered several miRNAs in human ES cells that overlapped with those detected in mouse ES cells, and also with those that are differentially expressed between self-renewing and differentiating daughter cells ${ }^{20}$. The development of this technology has significantly increased our ability to discover new miRNAs and other small RNAs, especially those with low expression levels or with modest differences in expression levels between various stages of stem cell differentiation ${ }^{21}$. It has to be noted that in conventional cloning and sequencing, miRNAs that are abundantly expressed in a cell are preferentially cloned and exclude those with low expression levels but possible significant functions in stem cells. Other deep-sequencing technologies, such as massively parallel signature sequencing ${ }^{22}$ and the Roche/454 platform ${ }^{12,23,24}$ (BOX 2), are also ready to use to identify a plethora of small RNAs in ES cells and various other tissues.

There are two issues that need to be addressed regarding the profiling of miRNA expression in relation to ES cell self-renewal and differentiation. First, individual miRNAs can show dynamic changes during stem cell differentiation, and therefore cloning and sequencing of miRNAs before and after differentiation would only allow us to assess ultimate changes, thereby precluding the assessment of dynamic changes of miRNAs during differentiation. This could be resolved by examining the expression of miRNAs at multiple time points during the course of stem cell differentiation. Second, the varying origin of ES cell lines and the heterogeneity of differentiated cells can skew and/or mask the true expression profile of miRNAs. This might have contributed to the varying results from different searches for ES cell-specific miRNAs. The recent application of single-cell real-time PCR to profile miRNA expression in primordial germ cells ${ }^{25}$ can be applied to evaluate the severity of this problem, as well as to help bypass it. In addition, homogeneous starting material and improved differentiation and cell selection protocols should also overcome the problem.

\section{Interplay between miRNAs and other regulatory pathways}

Research on ES cell-specific miRNAs has extended beyond their identification and phenotypic analysis, and is focusing on the regulation of their expression and their regulatory targets. These studies have in turn provided an opportunity to link miRNA regulation to other mechanisms of gene regulation in ES cells (FIG. 1). Although presently sporadic, this area of research should have a major role in integrating miRNA regulation to the biology of ES cells. 
A recent study shows that miR-21 (REF. 26), the levels of which increase following the induction of mouse ES cell differentiation, has potential binding sites in the $3^{\prime}$ UTRs of the mRNAs that encode for nanog, SOX2 and possibly OCT4. These are key proteins that are involved in ES cell self-renewal ${ }^{13}$. Other differentiation-related miRNAs - miR-134, miR-296 and miR-470 — were recently found to target coding regions of nanog, SOX2 and OCT4 to promote differentiation ${ }^{27}$. Hence, differentiation-related ES cell-specific miRNAs seem to promote differentiation by acting inside these cells to reduce the cellular concentrations of key proteins involved in self-renewal. Thus, the role of these miRNAs in ES cells can be compared to a Trojan Horse. Interestingly, the transcription of miR-21 itself is regulated in ES cells by a transcriptional repressor called the RE1-silencing transcription factor (REST), which directly interacts with cis elements upstream of the miR-21 gene ${ }^{26}$. Furthermore, a recent genome-wide chrom atin immunoprecipitation coupled with DNA sequencing (ChIP-seq) study has shown, at high resolution, that key ES cell-specific transcription factors bind to promoters of ES cell-specific miRNAs and directly promote their expression ${ }^{28}$. These studies demonstrate the existence of a regulatory paradigm from a transcription factor to miRNA and to another transcription factor in promoting ES cell differentiation.

In addition to regulatory interactions with transcription factors, the miRNA pathway also interfaces with epigenetic mechanisms. A known example of this is the ES cell-specific miR-290-295 cluster, which also acts like a Trojan Horse. This cluster seems to promote ES cell differentiation by acting inside ES cells to downregulate key proteins that are involved in ES cell self-renewal. The miR-290-295 cluster achieves this role by stably targeting repressors of DNA methyl transferases (DNMTs), such as retinoblastoma like-2 (RBL2), which methylate $\mathrm{CpG}$ islands, leading to stable epigenetic repression of Oct4 transcription $^{29,30}$ (FIG. 1). In fact, the miR-290-295 cluster seems to be responsible for the requirement of Dicer in ES cell differentiation. However, it remains to be addressed whether and how this epigenetic repression of Oct4 transcription is sufficient to promote differentiation, given that DNMTs are global regulators of $\mathrm{CpG}$ island methylation status in the entire genome.

Another important level of regulation of miRNA function resides at the level of miRNA biogenesis. This is shown by the regulation of the processing of the let-7 family of miRNAs by LIN28, a developmentally regulated RNA-binding protein that is involved in miRNA processing and promotes the reprogramming of human somatic cells into induced pluripotent stem (iPS) cells ${ }^{31}$. LIN28 selectively blocks the processing of pri-let-7 miRNAs in ES cells by specifically binding to conserved nucleotides in the loop region of the let-7 precursor and inhibiting the activity of Drosha and Dicer, thus having a central role in blocking miRNA-mediated differentiation in ES cells ${ }^{32,33}$. These findings also establish a novel role for the miRNA-precursor loop region in the regulated production of mature let-7 (REF. 34). These discoveries have started to reveal the intricate and complex web of regulatory networks through which miRNAs become part of the integrated mechanism of gene regulation that defines ES cell fate and behaviour. 


\section{miRNA function in adult stem cells}

The functions of miRNAs in somatic tissue stem cells have also been increasingly explored. This section reviews the miRNAs that have been identified in various somatic tissues and discusses how they regulate adult stem cell proliferation and differentiation.

\section{miRNAs regulate multiple steps of haematopoiesis}

Haematopoiesis is initiated by self-renewing divisions of haematopoietic stem cells, which give rise to complex cell types of myeloid and lymphoid lineages (FIG. 2). A combined approach that involves assessment of miRNA expression using microarray and bioinformatic prediction of mRNA targets revealed that distinct miRNA signatures fine-tune each step of haematopoiesis. For example, miR-128 and miR-181 are expressed only in early progenitor cells and prevent the differentiation of all haematopoietic lineages ${ }^{35}$. Likewise, miR-16, miR-103 and miR-107 prevent proliferation of later progenitor cells, whereas miR-221, miR-222 and miR-223 control the terminal differentiation pathways ${ }^{35}$ (FIG. 2).

The first lineage-specific decision during haematopoiesis is at the differentiation of common haematopoietic progenitor cells into common lymphoid or common myeloid progenitors. miR-223 and miR-181 are expressed less abundantly in the progenitor cells but are upregulated following differentiation into the myeloid and lymphoid lineages, respectively ${ }^{36}$. Largely consistent with this finding, ectopic expression of miR-181 in undifferentiated progenitor cells increases the population of B lymphoid lineage cells but not of myeloid lineage cells.

Within the myeloid lineage, several key mechanisms drive erythropoiesis. Bioinformatics analysis has suggested that miR-150, miR-155, miR-221 and miR-222 are progressively downregulated during erythropoiesis, whereas miR-451 and miR-16 are upregulated at late stages of erythropoiesis ${ }^{37}$. This implicates their function in these stages of erythropoiesis. Erythroid lineage differentiation is prevented by the repression of type I activin receptor (ALK4; also known as ACVR1B) by miR-24 (REF. 38). The growth factor activin, which, along with erythropoietin, promotes erythroid differentiation, binds to ALK4, resulting in a cascade of downstream signalling that culminates in erythroid differentiation. Another mechanism that prevents erythropoiesis involves miR-221 and miR-222, which block erythropoiesis by targeting KIT (also known as $C D 117$ ), a cytokine receptor that is expressed on the surface of haematopoietic stem cells ${ }^{39}$.

Monocytopoiesis is regulated by miRNA-17-5p, miR-20a and miR-106a, which target the monocytic differential transcription factor acute myeloid leukaemia 1 (AML1; also known as RUNX1) ${ }^{40}$. AML1 promotes monocytic differentiation by activating transcription of macrophage colony-stimulating factor (M-CSF), which is essential for monocytic differentiation.

Within the lymphoid lineage, the differential expression of miR-150 regulates lineage decision between $\mathrm{T}$ cells and $\mathrm{B}$ cells ${ }^{41}$. Ectopic expression of miR-150 in the lymphoid 
progenitor cells predisposes them to form more $\mathrm{T}$ cells than $\mathrm{B}$ cells. This might be achieved by targeting mRNAs that are responsible for B cell maturation.

As in ES cells, lineage-specific transcription factors can indirectly determine the fate of adult stem cells by modulating the levels of lineage-specific miRNAs. For example, the haematopoietic transcription factor GATA1 is required for the expression of miR-144 and miR-451, which are specifically induced during erythroid differentiation ${ }^{42}$. GATA1 interacts with its cofactor FOG1 (also known as ZFPM1), and brings about transcriptional activation by binding to sequence-specific elements that are distal to the miRNA gene that produces both miR-144 and miR-451 (REF. 42). In parental erythroblast cells that lack GATA1, GATA2 occupies the site but does not activate transcription, and thereby prevents erythroid differentiation. In another study, PU.1, a transcription factor that is essential for monocytic differentiation, activates miR-424. In turn, this targets the transcription factor nuclear factor I/A (NFI-A) and induces monocytic differentiation ${ }^{43}$. These observations reveal specific transcription factors as upstream regulators of the miRNA pathway in cell fate regulation.

\section{miRNAs in myogenesis and cardiogenesis}

Myogenesis is also a highly regulated proliferation and differentiation process, in which the mesodermal progenitor cells give rise to myoblasts that later withdraw from the cell cycle and fuse to form multinucleated myotubes ${ }^{44}$. Recent reports have revealed several mechanisms that are mediated by miRNAs during myogenesis. miR-1 and miR-133 are cotranscribed and processed specifically in adult cardiac and skeletal muscle tissues, but promote myogenesis in distinct fashions ${ }^{45}$. miR-1 indirectly upregulates myocyte enhancer factor 2C (MEF2C), an essential muscle-related transcription factor, by targeting the transcriptional co-repressor histone deacetylase 4 (HDAC4) and thereby promoting myogenesis. However, miR-133 targets serum response factor (SRF), a transcription factor that is essential for muscle proliferation and differentiation ${ }^{45}$. In another mechanism, miR-1 and miR-206 promote myogenesis by targeting connexin 43 (Cx43; also known as Gjal) mRNA and tightly regulating its protein levels ${ }^{46,47}$. CX43, which forms the gap junctions, is essential for normal skeletal muscle differentiation, and it is thought that gap junctions promote better intercellular transfer of the signals that are involved in myogenesis. In mouse and human ES cells, miR-1 and miR-133 both promote mesoderm formation and are potent repressors of non-muscle gene expression. However, they have opposing functions during the further differentiation of the mesodermal precursors into cardiac muscle progenitors: miR-1 promotes cardiac progenitor formation, whereas miR-133 represses the process ${ }^{48}$. The effect of miR-1 in promoting the mesodermal differentiation of ES cells seems to be partially achieved by translational repression of the notch ligand delta-like 1 (DLL1), which in turn promotes the expression of cardiac mesoderm genes and suppresses the expression of non-mesoderm genes ${ }^{48}$. These findings indicate that muscle-specific miRNAs reinforce the silencing of non-muscle genes during cell lineage commitment from pluripotent ES cells.

Another miRNA, miR-26a, indirectly promotes skeletal muscle differentiation by targeting the histone methyl-transferase enhancer of zeste homologue $2(\mathrm{EZH} 2)^{49}$. In undifferentiated mouse myoblasts, EZH2, along with the transcriptional regulators yin yang 1 (YY1) and 
HDAC1, epigenetically silences muscle-specific genes, and, following the induction of differentiation, EZH2 dissociates from muscle-specific genes. This paves the way for transcriptional activation of these genes ${ }^{50}$.

Similar to ES cells and haematopoiesis, muscle-specific transcription factors and miRNAs form a network to regulate myogenesis. For example, during cardiogenesis, SRF and the coactivator myocardin bind to cis-acting elements upstream of the genes that encode miR-1-1 and miR-1-2, and increase their cellular levels in cardiac progenitor cells ${ }^{51}$. These miRNAs in turn target HAND2, a transcription factor that is involved in ventricular cardiomyocyte expansion (proliferation), and help progenitor cells to choose between proliferation and differentiation. In another case, the myogenic factors myogenin and MYOD bind to cis elements upstream of loci that encode miR-1, miR-1-1, miR-1-2, miR-133, miR-133a-1 and miR-133a-2, and regulate their expression ${ }^{52}$.

Regulation of myogenesis also involves transforming growth factor- $\beta$ (TGF $\beta$ )-mediated downregulation of miR-24. This involves the direct binding of SMAD3 and SMAD4 to the promoter region of the miR-24 gene and regulating its expression ${ }^{53}$. Reducing miR-24 expression leads to the downregulation of myogenic differentiation markers in the $\mathrm{C} 2 \mathrm{C} 12$ myoblast cell line, whereas ectopic expression of miR-24-enhanced differentiation and partially rescued TGF $\beta$-inhibited myogenesis ${ }^{53}$. These results demonstrate a crucial role for miRNAs in modulating TGF $\beta$-dependent inhibition of myogenesis.

\section{miRNAs specify astrocyte versus neuronal fates}

Neuronal stem cells (NSCs) have to make crucial lineage-specific decisions as to whether to self-renew or differentiate into neurons or astrocytes ${ }^{54}$. As in other tissues, the differential expression of distinct miRNAs following the induction of neuronal differentiation dictates lineage-specific decisions. For example, miR-124 and miR-128 are specific to the neuronal lineage, whereas miR-26, miR-29 and miR-23 are preferentially and specifically expressed in astrocyte lineages respectively ${ }^{55}$. Consistent with these findings, overexpression of miR-124 and miR-128 in the NSCs prevents astrocyte differentiation and predisposes them towards neuronal differentiation ${ }^{56}$. miR-124 targets the $3^{\prime}$ UTR of small carboxy-terminal domain phosphatase 1 (SCP1; also known as CTDSP1) and induces neurogenesis ${ }^{57}$. SCP1 is an anti-neural factor that binds to a conserved response element (RE1) and prevents the expression of neural genes in non-neural tissues ${ }^{58}$. These studies show that miRNAs regulate the fate of neuronal stem cells by targeting key regulatory factors.

\section{miRNAs prevent osteogenic differentiation}

The function of miRNA in osteogenesis (bone formation) is now emerging. Osteoblasts (bone-forming cells) originate from multipotent mesenchymal stem cells, which differentiate into osteoblasts following induction by BMPs ${ }^{59}$. Two recent reports have revealed that miR-125b and miR-26a prevent the differentiation of mesenchymal stem cells into osteoblasts in distinct ways. miR-125b functions by inhibiting cell proliferation through an unknown mechanism ${ }^{60}$, whereas miR-26a prevents human adipose tissue-derived stem cells (ADSCs) from osteogenic differentiation by downregulating the translation of SMAD, a transcription factor that is required for late osteoblast differentiation ${ }^{61}$. These two studies 
support the role of miRNAs in maintaining the fate of osteogenic stem cells and progenitor cells.

\section{miRNAs promote differentiation in skin}

In contrast to osteogenesis, miRNAs in the skin have only been found to promote skin stem cell differentiation. Multipotent skin stem cells reside in the basal layer of the epidermis. They commit to the terminal differentiation pathway as they become suprabasal. In mouse epidermis, DICER1 is required for the maintenance of morphogenesis and for the maintenance of hair follicles. However, it was found not to be essential for skin stem cell fate determination and differentiation ${ }^{62,63}$. Furthermore, miR-203 was found to promote skin differentiation by limiting the proliferative capacity of the skin stem cells and induce cell cycle exit ${ }^{64}$. miR-203 is differentially expressed, with no expression in proliferative stem and progenitor cells but high expression in differentiated cells. A possible mechanism of miR-203 action is to target p63, which is an essential regulator of stem cell maintenance, although the mechanisms of action of p63 in stem cells are unclear.

\section{miRNAs and piRNAs in the germ line}

Germline stem cells are derived from primordial germ cells (PGCs) after they migrate into the embryonic gonad. In mammalian systems, only PGCs that migrate to the testicular cords on the male gonad become germline stem cells. During postnatal development, male germline stem cells undergo self-renewing divisions to produce many spermatogonia, which differentiate into spermatocytes, undergo meiotic divisions and eventually become mature sperm. By contrast, PGCs in the female gonad enter meiotic arrest before resuming meiosis during puberty.

Recent evidence suggests that, as in ES cells and somatic stem cells, the miRNA pathway has a vital role in both PGC specification and germline stem cell maintenance in invertebrates, and germline stem cell maintenance in vertebrates. In D. melanogaster, mature miRNAs are generated by a protein complex containing Dicer1 and Loquacious, a double-stranded RNA-binding protein. Depletion of maternal Dicer1 severely affects PGC formation in the early embryos ${ }^{65}$. In addition, knocking out either of these proteins ${ }^{66,67}$ affects female germline stem cell maintenance. Dicer1-null germline stem cells display delayed G1-S-phase transition ${ }^{68}$, which is similar to the phenotype displayed by Dicer-null ES cells. Mosaic analysis shows that the miRNA pathway might function intrinsically within the germline stem cells ${ }^{66,67}$. A similar role of Dicer1 for germline maintenance has also been shown in Caenorhabditis elegans ${ }^{69}$, which indicates that the function of the miRNA pathway in the germ line might be evolutionarily conserved.

In addition to miRNAs, the germ line has recently been found to be highly enriched for piRNAs that bind to Piwi-subfamily proteins ${ }^{11}$. Since Piwi proteins are required for germline development, germline stem cell self-renewal and gametogenesis ${ }^{70}$, piRNAs as Piwi partners might also be involved in germline stem cell functions. In support of this, a sub-telomeric piRNA in D. melanogaster has been correlated to germline stem cell maintenance ${ }^{12}$. In addition, piRNA-like ( $\left.32 \mathrm{nt}\right)$ small RNAs have been found in neoblasts (stem cells) of the planarian Schmidtea mediterranea ${ }^{71}$, which hints at a possibility that 
piRNAs might also be found in the stem cells of higher organisms. It will be interesting to see if mammalian piRNAs are also involved in these processes. Since Piwi and its associated piRNAs in D. melanogaster have also been shown to be involved in epigenetic regulation ${ }^{12}$, piRNAs might provide a new mechanism of regulation of the stem cell fate in the germ line through epigenetic programming.

\section{Conclusion and future perspectives}

It is becoming increasingly evident that miRNAs have crucial roles in the self-renewal and differentiation of all three kinds of stem cells - ES cells, somatic tissue stem cells and germline stem cells. Although research on the role of miRNAs in stem cells is still in its infancy, several general conclusions can already be drawn from current studies for miRNA function in stem cells. First, the miRNA pathway has an important role in somatic stem cells in diverse tissues, although it is unclear whether this role is achieved by fine-tuning the expression of mRNA targets, as expected from the mode of miRNA action, or by a more drastic impact on the expression of the target mRNAs. Second, stem cell self-renewal and differentiation are mediated by distinct miRNAs that are differentially expressed in a celltype- and stage-specific manner, although individual miRNAs can act at different stages of the stem cell lineage with multiple functions in promoting or repressing differentiation. Third, distinct profiles of miRNA expression in specific types of cells or during specific stages of differentiation are generated by conserved clusters of miRNAs that are coordinately transcribed, which in turn might function as master regulators of stem cell functions. Fourth, the regulatory function of the miRNA is often executed through, and controlled by, various transcription factors and other regulatory mechanisms of gene expression. These complex interactions between miRNAs and other regulators of gene expression at the epigenetic, transcriptional and post-transcriptional levels integrate miRNAs into the cellular network of regulation of gene expression that defines the stem cell fate and behaviour. Last but not least, the above modes of miRNA-mediated regulation seem to be well conserved among ES cells, various types of somatic tissue stem cells and germline stem cells. In fact, these might be the general modes of miRNA-mediated gene regulation during development.

miRNA functions in stem cells are being revealed at an extremely rapid rate. Further miRNAs will soon be discovered for their function in diverse stem cell systems by powerful deep-sequencing methods, combined with more effective methods for the isolation of stem cells and their differentiated progeny from various tissues. The mRNA targets of these miRNAs will be systematically identified by integrated experimental and bioinformatics approaches, and the effect of miRNAs towards their target mRNAs will be comprehensively assessed. The cellular effect of proteins translated from these target miRNAs will also be effectively measured. Equally importantly, the interplay between miRNAs and other cellular gene regulatory mechanisms, including other small RNA pathways (of which little is known thus far), will be systematically unravelled. These advances will not only lead to our indepth understanding of how miRNA-mediated gene regulation contributes to the fate and behaviour of stem cells, but should also shed light on the mechanism through which miRNAs regulate developmental processes in general. 


\section{Acknowledgments}

We thank members of the Lin laboratory for their valuable comments on the manuscript. We apologize to those whose works are not cited here owing to space limitations. The stem cell work done in the Lin laboratory is supported by National Institutes of Health Grants HD33760, HD37760S1 and HD42042, the Connecticut Stem Cell Research Fund, the G. Harold and Leila Mathers Foundation and the Stem Cell Research Foundation.

\section{Glossary}

Blastocyst

Niche

Spliceosome

Piwi

Transposon

Gap junction

Neoblast
An early stage of embryonic development at which cells begin to commit to two developmental lineages: the inner cell mass, which gives rise to the fetus, and the trophoblast, which gives rise to fetal support tissues, such as the placenta and the umbilical cord

The natural anatomical microenvironment that supports stem cell behaviour

A ribonucleoprotein (RNP) complex that is involved in splicing of nuclear pre-mRNA. It is composed of five small nuclear (sn) RNPs and more than 50 non-snRNPs, which recognize and assemble on exon-intron boundaries to catalyse intron processing of the pre-mRNA

An Argonaute or Piwi protein family member in Drosophila melanogaster that is required for germline stem cell self-renewal and also binds to 25 nucleotide small RNAs. Piwi is the founding member that was used to define the protein family

A mobile genetic element that can relocate within the genome of its host. An autonomous transposon encodes a transposase protein that catalyses its excision and reintegration in the genome, and can therefore direct its own transposition

An intercellular connection that directly connects cytoplasm of two cells so that exchange of molecules and ions can occur freely

An undifferentiated cell in annelids that proliferates to produce differentiated cells at the sites of repair

\section{References}

1. Sell, S. Stem Cells Handbook. Humana; Totowa, New Jersey: 2003.

2. Lin H. Cell biology of stem cells: an enigma of asymmetry and self-renewal. J Cell Biol. 2008; 180:257-260. [PubMed: 18227277]

3. Lin H. The stem-cell niche theory: lessons from flies. Nature Rev Genet. 2002; 3:931-940. [PubMed: 12459723]

4. Blakaj A, Lin H. Piecing together the mosaic of early mammalian development through microRNAs. J Biol Chem. 2008; 283:9505-9508. [PubMed: 18272516]

5. Rana TM. Illuminating the silence: understanding the structure and function of small RNAs. Nature Rev Mol Cell Biol. 2007; 8:23-36. [PubMed: 17183358]

6. Vasudevan S, Tong Y, Steitz JA. Switching from repression to activation: microRNAs can upregulate translation. Science. 2007; 318:1931-1934. Provides the first evidence for translational activation by miRNAs. [PubMed: 18048652] 
7. Okamura K, Hagen JW, Duan H, Tyler DM, Lai EC. The mirtron pathway generates microRNAclass regulatory RNAs in Drosophila. Cell. 2007; 130:89-100. [PubMed: 17599402]

8. Ruby JG, Jan CH, Bartel DP. Intronic microRNA precursors that bypass Drosha processing. Nature. 2007; 448:83-86. [PubMed: 17589500]

9. Babiarz JE, Ruby JG, Wang Y, Bartel DP, Blelloch R. Mouse ES cells express endogenous shRNAs, siRNAs, and other Microprocessor-independent, Dicer-dependent small RNAs. Genes Dev. 2008; 22:2773-2785. References 7-9 introduce new pathways for miRNA biogenesis. Reference 9 also shows that endogenous siRNAs are expressed in mouse ES cells. [PubMed: 18923076]

10. Okamura K, Lai EC. Endogenous small interfering RNAs in animals. Nature Rev Mol Cell Biol. 2008; 9:673-678. [PubMed: 18719707]

11. Lin H. piRNAs in the germ line. Science. 2007; 316:397. [PubMed: 17446387]

12. Yin H, Lin H. An epigenetic activation role of Piwi and a Piwi-associated piRNA in Drosophila melanogaster. Nature. 2007; 450:304-308. Provides the first evidence that piRNAs mediate epigenetic activation. [PubMed: 17952056]

13. Houbaviy HB, Murray MF, Sharp PA. Embryonic stem cell-specific microRNAs. Dev Cell. 2003; 5:351-358. Provides the earliest evidence for the existence of ES cell-specific miRNAs and also evidence that self-renewing and differentiating ES cells have distinct miRNAs. [PubMed: 12919684]

14. Tomari Y, Zamore PD. Perspective: machines for RNAi. Genes Dev. 2005; 19:517-529. [PubMed: 15741316]

15. Bernstein E, et al. Dicer is essential for mouse development. Nature Genet. 2003; 35:215-217. [PubMed: 14528307]

16. Kanellopoulou C, et al. Dicer-deficient mouse embryonic stem cells are defective in differentiation and centromeric silencing. Genes Dev. 2005; 19:489-501. References 15 and 16 describe the biological function of Dicer in mouse embryonic stem cells. [PubMed: 15713842]

17. Calabrese JM, Seila AC, Yeo GW, Sharp PA. RNA sequence analysis defines Dicer's role in mouse embryonic stem cells. Proc Natl Acad Sci USA. 2007; 104:18097-18102. [PubMed: 17989215]

18. Wang Y, Medvid R, Melton C, Jaenisch R, Blelloch R. DGCR8 is essential for microRNA biogenesis and silencing of embryonic stem cell self-renewal. Nature Genet. 2007; 39:380-385. Shows the specific requirement of the miRNA pathway for stem cell differentiation and cell cycle control. [PubMed: 17259983]

19. Suh MR, et al. Human embryonic stem cells express a unique set of microRNAs. Dev Biol. 2004; 270:488-498. This reference, along with reference 10, provides evidence for distinct miRNA signatures for self-renewing and differentiating stem cells. [PubMed: 15183728]

20. Morin RD, et al. Application of massively parallel sequencing to microRNA profiling and discovery in human embryonic stem cells. Genome Res. 2008; 18:610-621. [PubMed: 18285502]

21. Neilson JR, Zheng GX, Burge CB, Sharp PA. Dynamic regulation of miRNA expression in ordered stages of cellular development. Genes Dev. 2007; 21:578-589. [PubMed: 17344418]

22. Nakano M, et al. Plant MPSS databases: signature-based transcriptional resources for analyses of mRNA and small RNA. Nucleic Acids Res. 2006; 34:D731-D735. [PubMed: 16381968]

23. Margulies M, et al. Genome sequencing in microfabricated high-density picolitre reactors. Nature. 2005; 437:376-380. [PubMed: 16056220]

24. Hafner M, et al. Identification of microRNAs and other small regulatory RNAs using cDNA library sequencing. Methods. 2008; 44:3-12. [PubMed: 18158127]

25. Hayashi K, et al. MicroRNA biogenesis is required for mouse primordial germ cell development and spermatogenesis. PLoS ONE. 2008; 3:e1738. [PubMed: 18320056]

26. Singh SK, Kagalwala MN, Parker-Thornburg J, Adams H, Majumder S. REST maintains selfrenewal and pluripotency of embryonic stem cells. Nature. 2008; 453:223-227. [PubMed: 18362916]

27. Tay Y, Zhang J, Thomson AM, Lim B, Rigoutsos I. MicroRNAs to Nanog, Oct4 and Sox2 coding regions modulate embryonic stem cell differentiation. Nature. 2008; 455:1124-1128. Shows, for 
the first time, that miRNAs can also target coding regions instead of the $3^{\prime}$ UTR, and regulate translation of cognate mRNAs. [PubMed: 18806776]

28. Marson A, et al. Connecting microRNA genes to the core transcriptional regulatory circuitry of embryonic stem cells. Cell. 2008; 134:521-533. Shows, on a global scale, that key ES cell-specific transcription factors directly associate with promoter regions of ES cell-specific miRNAs, and thereby provides a mechanistic link between transcription factors and miRNA-dependent ES cell regulation. [PubMed: 18692474]

29. Benetti R, et al. A mammalian microRNA cluster controls DNA methylation and telomere recombination via Rbl2-dependent regulation of DNA methyltransferases. Nature Struct Biol. 2008; 15:268-279.

30. Sinkkonen L, et al. MicroRNAs control de novo DNA methylation through regulation of transcriptional repressors in mouse embryonic stem cells. Nature Struct Biol. 2008; 15:259-267.

31. Yu J, et al. Induced pluripotent stem cell lines derived from human somatic cells. Science. 2007; 318:1917-1920. [PubMed: 18029452]

32. Viswanathan SR, Daley GQ, Gregory RI. Selective blockade of microRNA processing by Lin28. Science. 2008; 320:97-100. [PubMed: 18292307]

33. Rybak A, et al. A feedback loop comprising lin-28 and let-7 controls pre-let-7 maturation during neural stem-cell commitment. Nature Cell Biol. 2008; 10:987-993. [PubMed: 18604195]

34. Newman MA, Thomson JM, Hammond SM. Lin-28 interaction with the Let-7 precursor loop mediates regulated microRNA processing. RNA. 2008; 14:1539-1549. [PubMed: 18566191]

35. Georgantas RW 3rd, et al. CD34 ${ }^{+}$hematopoietic stem-progenitor cell microRNA expression and function: a circuit diagram of differentiation control. Proc Natl Acad Sci USA. 2007; 104:2750 2755. [PubMed: 17293455]

36. Chen CZ, Li L, Lodish HF, Bartel DP. MicroRNAs modulate hematopoietic lineage differentiation. Science. 2004; 303:83-86. Provides the first evidence that miRNAs are involved in the differentiation of an adult stem cell lineage. [PubMed: 14657504]

37. Bruchova H, Yoon D, Agarwal AM, Mendell J, Prchal JT. Regulated expression of microRNAs in normal and polycythemia vera erythropoiesis. Exp Hematol. 2007; 35:1657-1667. [PubMed: 17976518]

38. Wang Q, et al. MicroRNA miR-24 inhibits erythropoiesis by targeting activin type I receptor ALK4. Blood. 2008; 111:588-595. [PubMed: 17906079]

39. Felli N, et al. MicroRNAs 221 and 222 inhibit normal erythropoiesis and erythroleukemic cell growth via kit receptor down-modulation. Proc Natl Acad Sci USA. 2005; 102:18081-18086. [PubMed: 16330772]

40. Fontana L, et al. MicroRNAs 17-5p-20a-106a control monocytopoiesis through AML1 targeting and M-CSF receptor upregulation. Nature Cell Biol. 2007; 9:775-787. [PubMed: 17589498]

41. Zhou B, Wang S, Mayr C, Bartel DP, Lodish HF. miR-150, a microRNA expressed in mature B and T cells, blocks early B cell development when expressed prematurely. Proc Natl Acad Sci USA. 2007; 104:7080-7085. [PubMed: 17438277]

42. Dore LC, et al. A GATA-1-regulated microRNA locus essential for erythropoiesis. Proc Natl Acad Sci USA. 2008; 105:3333-3338. [PubMed: 18303114]

43. Rosa A, et al. The interplay between the master transcription factor PU.1 and miR-424 regulates human monocyte/macrophage differentiation. Proc Natl Acad Sci USA. 2007; 104:19849-19854. [PubMed: 18056638]

44. Buckingham M. Myogenic progenitor cells and skeletal myogenesis in vertebrates. Curr Opin Genet Dev. 2006; 16:525-532. [PubMed: 16930987]

45. Chen JF, et al. The role of microRNA-1 and microRNA-133 in skeletal muscle proliferation and differentiation. Nature Genet. 2006; 38:228-233. [PubMed: 16380711]

46. Anderson C, Catoe H, Werner R. MIR-206 regulates connexin43 expression during skeletal muscle development. Nucleic Acids Res. 2006; 34:5863-5871. [PubMed: 17062625]

47. McCarthy JJ. MicroRNA-206: the skeletal muscle-specific myomiR. Biochim Biophys Acta. 2008; 1779:682-691. [PubMed: 18381085]

48. Ivey KN, et al. MicroRNA regulation of cell lineages in mouse and human embryonic stem cells. Cell Stem Cell. 2008; 2:219-229. [PubMed: 18371447] 
49. Wong CF, Tellam RL. MicroRNA-26a targets the histone methyltransferase enhancer of zeste homolog 2 during myogenesis. J Biol Chem. 2008; 283:9836-9843. [PubMed: 18281287]

50. Caretti G, Di Padova M, Micales B, Lyons GE, Sartorelli V. The polycomb Ezh2 methyltransferase regulates muscle gene expression and skeletal muscle differentiation. Genes Dev. 2004; 18:2627-2638. [PubMed: 15520282]

51. Zhao Y, Samal E, Srivastava D. Serum response factor regulates a muscle-specific microRNA that targets Hand2 during cardiogenesis. Nature. 2005; 436:214-220. [PubMed: 15951802]

52. Rao PK, Kumar RM, Farkhondeh M, Baskerville S, Lodish HF. Myogenic factors that regulate expression of muscle-specific microRNAs. Proc Natl Acad Sci USA. 2006; 103:8721-8726. [PubMed: 16731620]

53. Sun Q, et al. Transforming growth factor- $\beta$-regulated $m i R$ - 24 promotes skeletal muscle differentiation. Nucleic Acids Res. 2008; 36:2690-2699. [PubMed: 18353861]

54. Shi Y, Sun G, Zhao C, Stewart R. Neural stem cell self-renewal. Crit Rev Oncol Hematol. 2008; 65:43-53. [PubMed: 17644000]

55. Smirnova L, et al. Regulation of miRNA expression during neural cell specification. Eur J Neurosci. 2005; 21:1469-1477. [PubMed: 15845075]

56. Krichevsky AM, Sonntag KC, Isacson O, Kosik KS. Specific microRNAs modulate embryonic stem cell-derived neurogenesis. Stem Cells. 2006; 24:857-864. [PubMed: 16357340]

57. Visvanathan J, Lee S, Lee B, Lee JW, Lee SK. The microRNA miR-124 antagonizes the antineural REST/SCP1 pathway during embryonic CNS development. Genes Dev. 2007; 21:744-749. [PubMed: 17403776]

58. Yeo M, et al. Small CTD phosphatases function in silencing neuronal gene expression. Science. 2005; 307:596-600. [PubMed: 15681389]

59. Heino TJ, Hentunen TA. Differentiation of osteoblasts and osteocytes from mesenchymal stem cells. Curr Stem Cell Res Ther. 2008; 3:131-145. [PubMed: 18473879]

60. Mizuno Y, et al. miR-125b inhibits osteoblastic differentiation by down-regulation of cell proliferation. Biochem Biophys Res Commun. 2008; 368:267-272. [PubMed: 18230348]

61. Luzi E, et al. Osteogenic differentiation of human adipose tissue-derived stem cells is modulated by the miR-26a targeting of the SMAD1 transcription factor. J Bone Miner Res. 2008; 23:287295. [PubMed: 18197755]

62. Andl T, et al. The miRNA-processing enzyme dicer is essential for the morphogenesis and maintenance of hair follicles. Curr Biol. 2006; 16:1041-1049. [PubMed: 16682203]

63. Yi R, et al. Morphogenesis in skin is governed by discrete sets of differentially expressed microRNAs. Nature Genet. 2006; 38:356-362. [PubMed: 16462742]

64. Yi R, Poy MN, Stoffel M, Fuchs E. A skin microRNA promotes differentiation by repressing 'stemness'. Nature. 2008; 452:225-229. [PubMed: 18311128]

65. Megosh HB, Cox DN, Campbell C, Lin H. The role of PIWI and the miRNA machinery in Drosophila germline determination. Curr Biol. 2006; 16:1884-1894. [PubMed: 16949822]

66. Park JK, Liu X, Strauss TJ, McKearin DM, Liu Q. The miRNA pathway intrinsically controls selfrenewal of Drosophila germline stem cells. Curr Biol. 2007; 17:533-538. [PubMed: 17320391]

67. Forstemann K, et al. Normal microRNA maturation and germ-line stem cell maintenance requires Loquacious, a double-stranded RNA-binding domain protein. PLoS Biol. 2005; 3:e236. [PubMed: 15918770]

68. Hatfield SD, et al. Stem cell division is regulated by the microRNA pathway. Nature. 2005; 435:974-978. [PubMed: 15944714]

69. Knight SW, Bass BL. A role for the RNase III enzyme DCR-1 in RNA interference and germ line development in Caenorhabditis elegans. Science. 2001; 293:2269-2271. [PubMed: 11486053]

70. Beyret, E.; Lin, H. MicroRNAs — from Basic Science to Disease Biology. Appasani, K., editor. Cambridge Univ. Press; New York: 2008. p. 497-511.

71. Palakodeti D, Smielewska M, Lu YC, Yeo GW, Graveley BR. The PIWI proteins SMEDWI-2 and SMEDWI-3 are required for stem cell function and piRNA expression in planarians. RNA. 2008; 14:1174-1186. [PubMed: 18456843] 


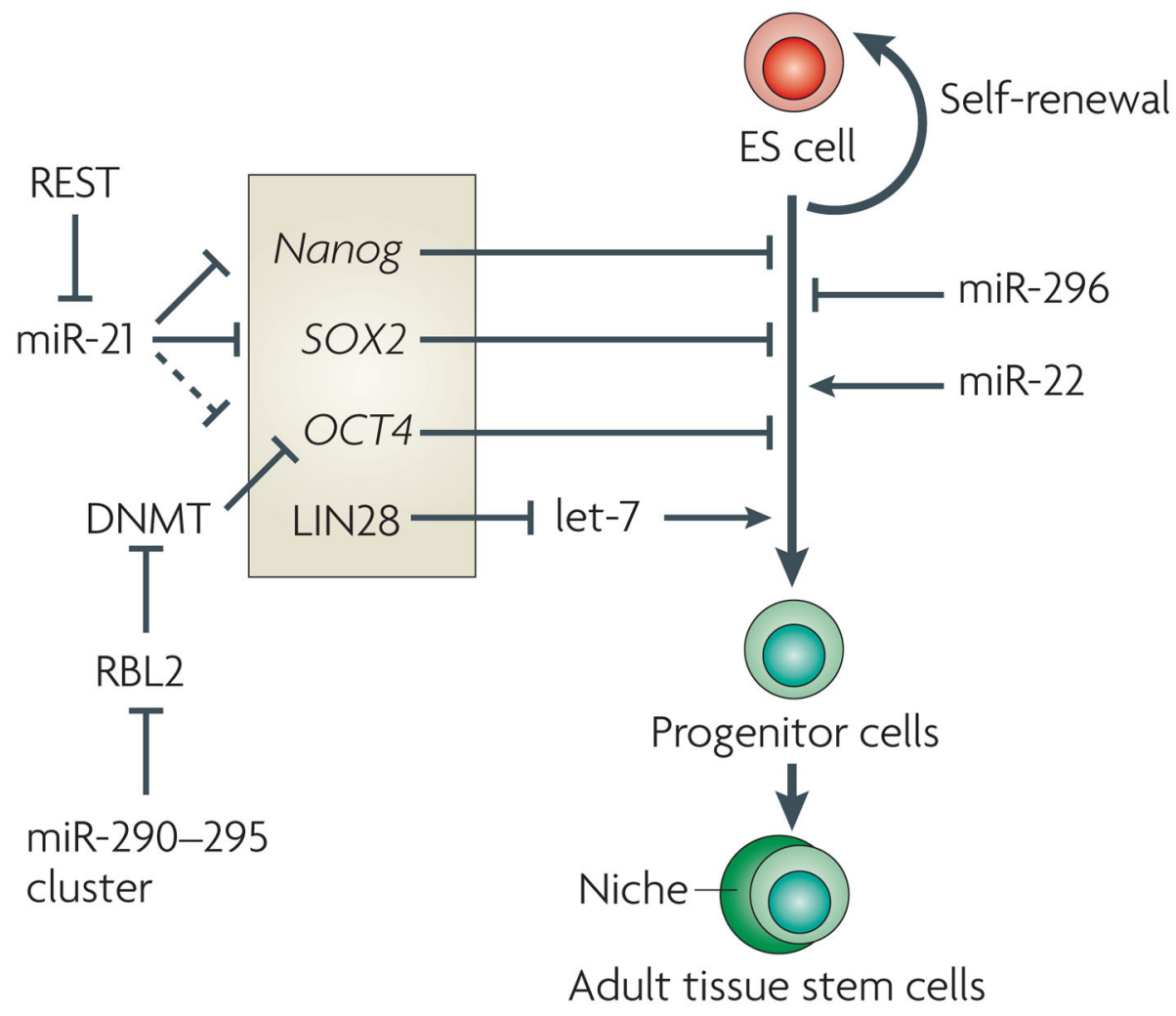

Figure 1. RNA regulation of embryonic stem cells

Embryonic stem (ES) cells have the ability to self-renew or differentiate into progenitor cells, which finally give rise to all of the adult tissue stem cells in the body. MicroRNAs (miRNAs) regulate both self-renewal and differentiation pathways of ES cells by regulating various factors that mediate these processes. miRNAs form an integral network with transcription factors in regulating stem cell processes. For example, RE1-silencing transcription factor (REST), a transcriptional repressor, downregulates miR-21, which targets Nanog, SOX2 and OCT4 (also known as OCT3 and POU5F1). These are essential for stem cell self-renewal. Therefore, REST promotes self-renewal ${ }^{26}$. Self-renewal is also promoted by the miR-290-295 cluster, which targets retinoblastoma like 2 (RBL2) - a repressor of DNA methyl transferases (DNMTs) — which methylate CpG islands and epigenetically silence OCT4 (REFS 29,30). Another mechanism of promoting self-renewal is by the regulation of let-7 miRNA processing by LIN28, which directly binds to a consensus sequence in the pre-let-7 miRNA loop region and inhibits processing by Drosha and Dicer ${ }^{32,33}$. miR-296 promotes ES cell differentiation and miR-22 inhibits ES cell differentiation. However, their mRNA targets have not been identified. Positive and negative regulations are shown by an arrow and a $\mathrm{T}$ line respectively; a dotted $\mathrm{T}$ line suggests a possible pathway that needs to be confirmed. 


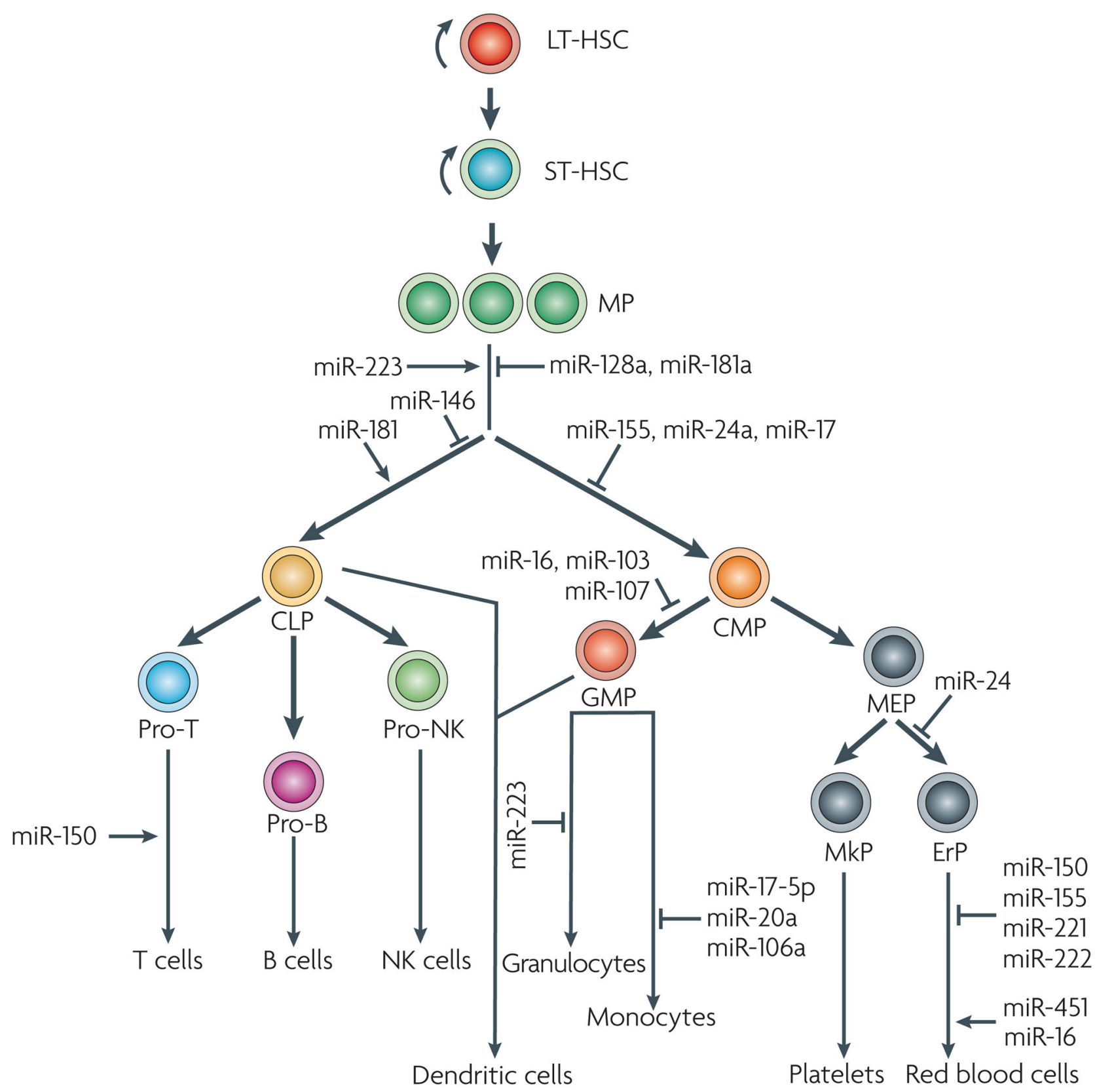

Figure 2. RNA regulation of haematopoietic stem cells

Haematopoiesis involves a cascade of cell proliferation and differentiation in which the long-term reconstituting haematopoietic stem cells (LT-HSCs) first give rise to short-term reconstituting HSCs (ST-HSCs), followed by multipotent progenitors (MPs). MPs differentiate into either common lymphoid progenitors (CLPs) or common myeloid progenitors (CMPs). CLPs and CMPs further differentiate to produce various cells of the bloodstream. A number of microRNAs (miRNAs) have been identified that fine-tune each step of the haematopoietic differentiation. miRNAs that function in specific steps are marked, and positive and negative regulations are illustrated by an arrow and a $\mathrm{T}$ line, 
respectively. ErP, erythroid progenitor; GMP, granulocyte-macrophage progenitor; MEP, megakaryocytic-erythroid progenitor; MkP, megakaryocyte progenitor; NK, natural killer. 


\section{Table 1}

RNAs identified in various kinds of stem cells

\begin{tabular}{|c|c|c|c|}
\hline Stem cell process & Cell type or lineage & miRNAs involved ${ }^{*}$ & Targets $\S$ \\
\hline \multicolumn{4}{|l|}{ ES cells } \\
\hline \multirow[t]{2}{*}{ Self-renewal } & Mouse ES cells & $\begin{array}{l}\text { miR-290-295 cluster, miR-296, miR-302 (REF. } 13 \text { ), } \\
\text { miR-17-92 cluster and miR-15b-16 } \text { cluster }^{17}\end{array}$ & No targets identified \\
\hline & Human ES cells & $\begin{array}{l}\text { miR-371, miR-372, } \mathrm{miR}-373 *-373, \mathrm{miR}-200 \mathrm{c} \\
\text { miR-368 and miR-154* (REF. 19) }\end{array}$ & No targets identified \\
\hline \multirow[t]{2}{*}{ Differentiation } & Mouse ES cells & miR-21 and miR-22 (REF. 13) & $\begin{array}{l}\text { miR-21 targets Nanog and Sox } 2 \\
\text { (REF. 26) }\end{array}$ \\
\hline & Human ES cells & $\begin{array}{l}\text { miR-301, miR-374, miR-21, miR-29b and miR-29 } \\
\text { (REF. 19) }\end{array}$ & No targets identified \\
\hline \multicolumn{4}{|l|}{ Somatic stem cells } \\
\hline \multirow[t]{7}{*}{ Haematopoiesis } & Progenitor cells & $\begin{array}{l}\text { miR-128, miR-181, miR-16, miR-103 and miR-107 } \\
\text { (REF. 35) }\end{array}$ & No targets identified \\
\hline & Pro-T lymphoid cells & miR-150 (REF. 41) & No targets identified \\
\hline & Pro-B lymphoid cells & $\begin{array}{l}\text { miR-181, miR-155, miR-24, miR-17, miR-16, } \\
\text { miR-103 and miR-107 (REF. 35) }\end{array}$ & No targets identified \\
\hline & Erythroid myeloid cells & $\begin{array}{l}\text { miR-150, miR-155, miR-221, miR-222, miR-451, } \\
\text { miR-16 (REFS 35,37) and miR-24 (REFS 35,38) }\end{array}$ & $\begin{array}{l}\text { miR-24 targets human } A L K 4 ; \text { and } \\
\text { miR-221 and miR-222 target human } \\
\text { KIT (REF. 39) }\end{array}$ \\
\hline & Monocytes & $\begin{array}{l}\text { miR-17-5p, miR-20a, miR-106a, miR-16, miR-103 } \\
\text { and miR-107 (REF. 40) }\end{array}$ & $\begin{array}{l}\text { miR-17-5p, miR-20a and miR-106a } \\
\text { target } A M L 1\end{array}$ \\
\hline & Granulocytes & $\begin{array}{l}\text { miRNA-155, miR-24, miR-17, miR-223, miR-16, } \\
\text { miR-103 and miR-107 (REF. 35) }\end{array}$ & No targets identified \\
\hline & Megakaryocytes & miR-155, miR-24, and miR-17 (REF. 35) & No targets identified \\
\hline \multirow[t]{2}{*}{ Myogenesis } & Skeletal cells & $\begin{array}{l}\text { miR-1, miR-133 (REF. 45), miR-206 (REF. 46) and } \\
\text { miR-26a (REF. 50) }\end{array}$ & $\begin{array}{l}\text { miR-1 targets mouse } H \text { dac } 4 \text {; miR-133 } \\
\text { targets mouse } \text { Srf; miR- } 1 \text { and } \\
\text { miR-206 target mouse connexin } 43 \\
\text { (also known as Gjal); and miR-26a } \\
\text { targets mouse Ezh2 }\end{array}$ \\
\hline & Cardiac cells & Unknown & Unknown \\
\hline \multirow[t]{2}{*}{ Neurogenesis } & Neuronal & miR-124 and miR-128 (REFS 55,56) & $\begin{array}{l}\text { miR-124 targets chicken SCP1 (REF. } \\
57 \text { ) }\end{array}$ \\
\hline & Astrocytes & miR-26, miR-29, miR-23 (REF. 55) & No targets identified \\
\hline Osteogenesis & Osteoblasts & $\operatorname{miR}-125 b^{60}$ and $\mathrm{miR}-26 \mathrm{a}$ & miR-26a targets human $S M A D$ \\
\hline Skin development & Keratinocytes & miR-203 (REF. 64) & miR-203 targets mouse $p 63$ \\
\hline
\end{tabular}

${ }^{*}$ Specific miRNAs have yet to be discovered in germline stem cells.

$\S_{\text {Only miRNAs with targets are shown. }}$

$A L K 4$, type I activin receptor; AML1, acute myeloid leukaemia 1; ES cell, embryonic stem cell; Ezh2, enhancer of zeste homologue 2; Hdac4, histone deacetylase 4; miRNA, microRNA; SCP1, small carboxy-terminal domain phosphatase 1; Srf, serum response factor. 